\title{
¿Qué Estructuras Deductivas Usan Alumnos Ingresantes a la Universidad?
}

\author{
Which Deductive Structures Are Used by Freshmen?
}

\author{
Eduardo Mario Lacues Apud* \\ ORCID iD 0000-0001-7449-999X \\ Leonora Díaz Moreno** \\ ORCID iD 0000-0001-5765-6332 \\ Juan Antonio Huertas Martinez*** \\ ORCID iD 0000-0002-1518-8398
}

\begin{abstract}
Resumen
En este trabajo se presentan resultados de una indagación que inquirió el efecto que, sobre estudiantes ingresantes a la universidad, tuvieron cursos universitarios iniciales de Matemática para adquirir conocimientos que faciliten la comprensión y el uso de estructuras deductivas de uso frecuente en razonamientos o argumentaciones. Este tema es de interés actual en Uruguay, debido a la preocupación que generan las altas tasas de rezago y abandono en primer año. Como antecedentes se efectuó un análisis de libros de texto usuales y de registros estudiantiles de clases, en busca de evidencias acerca del grado de explicitación de estas estructuras en la enseñanza. Entre los resultados observados destacan que los ingresantes muestran conocimientos acerca de las estructuras deductivas para comprender o construir razonamientos, y que la sola participación en cursos de Matemática, sin mediar una enseñanza intencional, no parece suficiente para adquirir habilidades en esta área.
\end{abstract}

Palabras clave: Estructura Lógica del Condicional. Estructuras Deductivas. Razonamiento. Matemática en la Universidad. Ingresantes a la universidad.

\begin{abstract}
This report presents the results of a research that inquired about the effects over freshmen of initial Mathematics courses at university have on the learning of contents which aims to the comprehension and use of deductive structures frequently used in reasoning or argumentations. This issue is interesting in Uruguay, because of students' high rates of desertion at first year. Antecedents to this study are an analysis of textbooks and classroom notes taken by students, in search of evidence about how explicit are these structures in teaching practices. Among the results observed, it may be highlighted that only the participation in Mathematics courses, in absence of intentional instruction, seems not to be enough to acquire skills on these subjects, and only few evidences

\footnotetext{
* Magister en Eduacación, Universidad Católica del Uruguay (UCU). Profesor de tiempo completo, Universidad Católica del Uruguay (UCU), Montevideo, Uruguay. Dirección postal: Av. 8 de octubre, 2738, Tres Cruces, Montevideo, Uruguay, C.P: 11600. E-mail: elacuees@ucu.edu.uy.

** Doctora en Ciencias de la Educación, Pontificia Universidad Católica de Chile (PUCCH). Profesora, Universidad de Valparaíso (UV), Valparaíso, V Región, Chile. Dirección postal: Av. Gran Bretaña, 1111, Playa Ancha, Valparaíso, V Región, Chile, C.P: 2340000. E-mail: leonora.diaz@uv.cl.

*** Doctor en Psicología. Universidad Autónoma de Madrid (UAM). Profesor Titular de Psicología Básica, Departamento de Psicología Básica, Facultad de Psicología, Universidad Autónoma de Madrid (UAM), Madrid, España. Dirección postal: Calle Ivan Pavlov, 4, C.P: 28049. E-mail: juanantonio.huertas@uam.es.
} 
were found that shows that teaching takes in consideration instruction in Logic, except in specific texts or courses.

Keywords: Conditional Logic Structure. Deductive Structures. Reasoning. College Mathematics. University Freshmen.

\section{Introducción}

Es frecuente que se asocie Matemática con razonamiento, y se señale esta vinculación como una de las razones para justificar su inclusión en el currículo. Inglis y Simpson (2008) rastrean esta idea hasta Thorndike y su Theory of Formal Discipline, al presentar hitos que, en el siglo XX, marcaron la evolución de la Matemática.

Si bien esta asociación es difusa, ya que no se distingue en ella qué clases de razonamiento se identifican con la Matemática, suele confiarse en que el solo aprendizaje de la disciplina alcanza para desarrollar habilidades de razonamiento. Esta postura ha sido cuestionada, entre otros, por Bloch (2003) al señalar que la enseñanza del Cálculo suele desarrollarse sin vincularla al de los procesos deductivos.

En el razonamiento deductivo, que juega un rol central dentro de los procesos de demostración, la estructura lógica del condicional es esencial. En la enseñanza de Matemática tiene una presencia importante, manifestada en el discurso por el uso de expresiones tales como: la propiedad tal es condición necesaria para la propiedad cual, la ocurrencia de tal implica la de cual, si tal entonces cual, alcanza con tal para que cual, entre muchas otras. Estas expresiones forman parte del habla en el aula, tanto oral como escrita, y de los estudiantes como de los profesores, y también de los libros de texto.

Sin embargo, hay evidencias que ilustran que diferentes actores no comprenden lo mismo al formular las mismas expresiones, lo que tiene implicaciones en la enseñanza y el aprendizaje (DURAND-GUERRIER, 2003; HODDS; ALCOCK; INGLIS, 2014). Un punto central es el de la adjudicación de valor de verdad a la estructura lógica del condicional, que fundamenta las formas de argumentación propias de la Matemática. Pero, como destacan Crespo, Farfán y Lezama (2010), no sólo éstas no son las únicas, sino que con frecuencia no son las usadas en el aula, ya que estudiantes o profesores utilizan otras.

En otro orden, se ha señalado (CAMACHO; SÁNCHEZ-POZOS; ZUBIETA, 2014) que los estudiantes de carreras de Ingeniería o Ciencias no tienen familiaridad con las nociones de demostración, rigor o formalidad, aún cuando no sean principiantes. En su trabajo, los autores se propusieron identificar los argumentos (y dentro de ellos, los que sean 
correctos) que utilizan estudiantes para justificar los pasos de demostraciones cuya lectura se les solicita. Entre sus conclusiones señalan que, frecuentemente, los estudiantes apelan a su intuición y al contenido involucrado para dar justificaciones, y no a las estructuras deductivas usadas. En el mismo sentido, apuntan las diferencias que reportan Inglis y Alcock (2012) entre expertos y novatos al leer demostraciones.

Por otro lado, reportes como los de Amado, Sanchez y Pinto (2015) ejemplifican actividades asistidas por software apropiado, que facilitan la manipulación de figuras y brindan oportunidades de formular y explorar conjeturas, lo que permite a los estudiantes aproximarse a la noción de demostración.

En un sentido concurrente, Morou y Kalospyros (2011) relatan el diseño de un curso de Lógica para enseñanza media, en el que entre otras características, se presentan materiales informales (como puzzles o situaciones tomadas de obras de ficción) para mostrar el uso de las formas de argumentación y demostración no sólo en contextos matemáticos. Entre sus conclusiones, destacan la necesidad de enseñar la noción de prueba:

[...] the transfer from the logic taught, such as first-order logic with quantifiers, to logic applied in understanding the deductive structure of mathematics and constructing proofs doesn't happen automatically. Yet many educators suggest students must be made familiar with the principles of mathematical argumentation, that is, they must be taught proof (MOROU; KALOSPYROS, 2011, p. 8) ${ }^{1}$

En este trabajo se informa un estudio de caso sobre el efecto de primeros cursos de Matemática, uno de Cálculo y otro de Lógica, en la modificación de las ideas de estudiantes ingresantes a la universidad, sobre el condicional y las estructuras deductivas. Dado que en el segundo se tratan, como contenidos curriculares, las nociones de condicional y de prueba lógica, mientras que éstos no figuran explícitamente en el primero, resulta de interés estudiar la existencia de eventuales diferencias en los efectos de cada curso.

Para inferir modos en que esas nociones se habrían tratado en su enseñanza, se examinaron como antecedentes los tratamientos de estos temas en libros de texto usuales en Uruguay, y apuntes de clase tomados por estudiantes participantes en este estudio, correspondientes a cursos de Matemática de su último año de bachillerato, el anterior a su ingreso a la universidad.

La primera sección trata del condicional y de las formas de presentación del

\footnotetext{
1، [...] la transferencia de la lógica enseñada, tal como lógica de primer orden con cuantificadores, a la lógica aplicada en comprender la estructura deductiva de Matemática y en construir pruebas no ocurre automáticamente. Así, muchos educadores sugieren que los estudiantes deben ser familiarizados con los principios de la argumentación matemática, esto es, se les debe enseñar demostraciones". Traducción de los autores.
} 
razonamiento deductivo a través de las reglas de inferencia, brindando un panorama histórico de la noción de condicional que destaca aspectos epistemológicos relevantes. La sección que sigue presenta el tratamiento que hacen de los temas de Lógica libros de texto usuales en los cursos elegidos y los análisis de registros estudiantiles de Matemática de bachillerato. Se continua, describiendo el estudio de caso: el diseño experimental, la forma en que se recogieron los datos y el análisis de los mismos. Se cierra presentando conclusiones y reflexiones finales.

\section{Trayecto Histórico de la Noción de Condicional y Reglas de Inferencia}

En Cálculo Proposicional, dadas dos fórmulas bien formadas (fbf) A y B se define el valor de verdad de $(\mathrm{A}) \rightarrow(\mathrm{B})$ poniendo que es falsa si y sólo si la fbf A es cierta y la fbf B es falsa. Las fbf A y B se llaman antecedente y consecuente, respectivamente, del condicional $(\mathrm{A}) \rightarrow(\mathrm{B})$.

Los orígenes de la definición del valor de verdad de esta fórmula (llamada implicación material por Bertrand Russell, a principios del siglo XX) pueden remontarse a Filón de Megara (DURAND-GUERRIER, 2003) cuyo trabajo se desarrolló alrededor del año 300 a.C., retomando y ampliando el de Aristóteles (384-322 a.C.); este último, al introducir los silogismos, había revelado una temprana preocupación por un aspecto no considerado hasta ese momento: la necesidad de cuantificar proposiciones.

Esta valoración de la implicación material trae como consecuencia la generación de aparentes paradojas: a partir de una premisa falsa puede obtenerse cualquier conclusión; dadas dos fórmulas, siempre una es implicada por la otra. Estos resultados, cuya demostración desde el punto de vista lógico no ofrecen dificultad, resultan anti-intuitivos. Posiblemente, una causa sean las prácticas deductivas usuales en Matemática que asumen que las hipótesis son ciertas, lo que parece generar la idea de la necesidad de que sean ciertas, como se discute a continuación.

Cualquier cadena deductiva se basa en reglas de inferencias, como el modus ponens, en el que se plantea que de una sentencia condicional que se asume cierta, si además se sabe cierto el antecedente, puede concluirse que el consecuente es cierto. Asumir que el antecedente es cierto (es decir, la hipótesis es cierta) no es necesario para que el consecuente sea cierto; sin embargo, para poder afirmarlo a partir de la regla, sí debe serlo. El uso reiterado de esta regla podría, por eso, inducir al convencimiento de que es suficiente analizar 
lo que ocurre en este caso.

Esta convicción puede resultar estimulada por una de las aplicaciones más frecuentes de las sentencias condicionales, la descripción de relaciones causales: "La concepción de los condicionales en los estudiantes suele ser causal..." (CRESPO; FARFÁN; LEZAMA, 2010, p. 267).

En efecto, las sentencias con forma condicional, usualmente, son interpretadas en el sentido de que el hecho indicado en el antecedente es la causa de lo que se señala en el consecuente. En esta interpretación, sólo tiene relevancia establecer, si una vez ocurrida la causa (es decir, si el antecedente es verdadero), ocurre o no el fenómeno descrito como eventual efecto (es decir, el consecuente es verdadero o es falso). En el primer caso, la sentencia expresa una relación correcta (es decir, se le asigna el valor cierto a la sentencia condicional) mientras que en el segundo la relación es incorrecta (o sea, la sentencia condicional es falsa).

En esta aplicación, es irrelevante la consideración de lo que pasa cuando el hecho pretendido como causa no ocurre (es decir, el antecedente toma el valor falso) dado que no aporta a la descripción del fenómeno que se quiere estudiar.

La aparición de una Lógica Matemática (KLINE, 1972) puede rastrearse hasta unos trabajos preliminares e incompletos de Descartes y, posteriormente, hasta Leibnitz, quien, hacia fines del siglo XVII, pretendió comenzar a desarrollar un álgebra lógica, logrando algunos resultados pero sin completar su propósito. Brunschvicg resalta el objetivo de Leibnitz de desarrollar una Simbólica o Característica, es decir, una forma de representación que sirviera como ámbito de razonamiento: "Es de esta ciencia que dependería entonces el desarrollo de las diferentes partes de la Matemática" (BRUNSCHVICG, 1945, p. 227).

En el mismo sentido, apunta la posición defendida por Peano (1895) al tratar la definición de límite de una función, manifestando que la lógica matemática tiene como objetivo el de representar todas las proposiciones matemáticas, utilizando el menor número posible de convenciones, para abreviar el trabajo de escritura y constituirse en un instrumento para el desarrollo del pensamiento.

En el siglo XIX, los trabajos de Augustus de Morgan y George Boole significan avances importantes. Siguiendo a Kline (1972), un aspecto relevante del trabajo del primero fue introducir la idea de cuantificación, señalando que los términos pueden ser cuantificados.

El trabajo de George Boole permitió algebrizar el Cálculo Proposicional, reduciendo el problema de calcular el valor de verdad de una fórmula al de encontrar el valor de una expresión mediante reglas que modernamente se han convertido en los axiomas que definen 
un Álgebra de Boole (GLYMOUR, 1997). Sin embargo, con ser importantísimo, el aporte de Boole no consiguió resolver el problema de manejar variables individuales y sus cuantificaciones.

Recién en el siglo XX, gracias a los trabajos de Frege, se pudo abordar este problema con éxito. Siguiendo a Glymour (1997), Frege, en desconocimiento de los avances de Boole, comenzó su programa consistente en reducir Matemática a Lógica. Su trabajo innovador introdujo las nociones de variable, función de símbolos, predicado de símbolos (o función proposicional) y cuantificador, creando lo que se conoce como Cálculo de Predicados. Estos avances permitieron formalizar la definición actual de implicación material.

Es importante destacar que, en muchas definiciones o demostraciones matemáticas, están presentes, a veces de manera implícita, instancias de cuantificación, lo que contribuye a las dificultades para su aprendizaje. Éste es el centro de la argumentación de Durand-Guerrier (2005) respecto de la necesidad de atender desde el punto de vista didáctico, a la presentación en términos de Cálculo de Predicados de las demostraciones en Matemática, y en particular, de los procesos de cuantificación que contienen.

Dada la relevancia de la noción de condicional en la actividad matemática, y las dificultades que debieron superarse hasta establecer de modo formal la implicación material, surge la cuestión de si la sola exposición de los estudiantes a la actividad matemática escolar alcanza para que se apropien de este concepto, o si para ello es necesario que éste sea objeto explícito de enseñanza.

\section{Motivación y Preguntas de Investigación}

Muchas de las demostraciones que se realizan en la enseñanza de Matemática se sustentan, casi exclusivamente, en un lenguaje vernáculo, aun cuando intervengan gráficas, diagramas, tablas u otras representaciones.

Las formas deductivas, si bien responden a una estructura lógica formal, se presentan en clase a través de expresiones como condición necesaria, condición suficiente, tener que, alcanzar con, implicar, ser consecuencia de, entre otras.

Es relevante preguntarse qué conocimientos presentan los ingresantes acerca del conectivo condicional (que da soporte a estas formas deductivas), y si estos conocimientos son modificados como consecuencia de sus primeros cursos en el área de Matemática.

En relación con este segundo punto, interesó indagar si, en caso de constatarse cambios, éstos eran independientes del curso en el que el estudiante hubiera participado. En 
particular, si un curso que incorpora la enseñanza explícita de temas de Lógica como parte de sus contenidos, se diferencia de otros, como el de Cálculo Diferencial, en este aspecto.

El estudio se organizó en dos partes complementarias que se describen a continuación.

\subsection{Antecedentes: registros de clase y textos}

Se seleccionaron dos fuentes documentales: apuntes de estudiantes (registrados en sus cursos de Matemática previos a la universidad) y textos de Cálculo o de Lógica de uso habitual en cursos universitarios.

En ambos casos, la revisión prestó atención a identificar las instancias en las que se efectuaban demostraciones o inferencias, en busca de indicaciones que explicitaran las estructuras deductivas utilizadas y de eventuales errores en el uso de ellas.

Tanto en el caso de los apuntes de estudiantes como en el de los textos de Cálculo, se eligió observar el tratamiento del tema de la definición y teoremas acerca del límite de una función en un punto, por la estructura lógica que los configuran (sentencias condicionales).

\subsubsection{Análisis de apuntes de estudiantes}

El análisis de los apuntes registrados por los estudiantes en sus clases, aunque con gran desarrollo en otras áreas, no ofrece muchos antecedentes para inferir acerca de la enseñanza de Matemática (ARCE; CONEJO; ORTEGA DEL RINCÓN, 2016). Ellos constatan en su investigación la coexistencia de diversos estilos de toma de notas de clase (exhaustivo, selectivo-estratégico, ausencia de relaciones), en un contexto donde los profesores suelen mostrar un estilo magistral de enseñanza.

En esta sección, se revisan los apuntes de clase tomados en un curso de Cálculo de bachillerato por ocho estudiantes, elegidos entre quienes participaron en los cursos de este estudio (33 estudiantes en total, como se detalla más adelante); se trata de casos polares, en tanto que cuatro tuvieron buenos resultados académicos en ellos, y otros cuatro registraron fracaso. Esta selección fue intencional, con el objetivo de hacer evidentes eventuales diferencias al contrastar los registros en revisión.

En general, los apuntes muestran poca evidencia de tratamiento de los temas elegidos. Al respecto, se encuentran antecedentes en Díaz (1999), que muestran una enseñanza que soslaya la construcción de significados de límite en cursos iniciales de Cálculo de programas de ingeniería para una población de más de dos mil estudiantes. Se recurre a protocolos 
esquemáticos de la demostración $\{\varepsilon, \delta\}$, eludiendo una enseñanza para los aprendizajes de la estructura lógica subyacente a esta entidad matemática.

En el caso de los estudiantes que experimentaron fracaso, los registros de clase casi no muestran referencias a elementos conceptuales (definiciones, teoremas). Se constata la preocupación por recopilar situaciones típicas de uso de algoritmos para resolver ejercicios, sin que estos procedimientos de cálculo sean justificados de algún modo. Un ejemplo de esto lo constituye el conjunto de reglas para calcular límites: no se encuentran referencias que liguen estos procedimientos con los teoremas que les dan soporte lógico.

Las notas de dos de los cuatro estudiantes con buenos resultados académicos reiteran estas características: no recogen referencias a estructuras deductivas; consisten, principalmente, en un conjunto de reglas de cálculo, enunciadas sin demostración alguna y utilizadas para resolver ejercicios.

Sin embargo, en el caso de los otros dos estudiantes de este grupo, se da una situación diferente.

En los registros del estudiante 1 se encuentra una transcripción precisa de la definición de límite, que usa luego en la demostración de diferentes teoremas acerca de límites. Dado que en la mayoría de estos teoremas el resultado se establece a partir de la prueba de que una cierta desigualdad se satisface en un cierto dominio para la variable, sus argumentaciones no plantean formas deductivas, sino la forma en la que las hipótesis se insertan en la desigualdad a verificar, transformando la demostración en un trabajo con inecuaciones.

Sin embargo, sí se nota preocupación por explicitar el rol que juegan los cuantificadores, en particular, para enfatizar que, dadas dos condiciones, que se satisfacen en respectivos entornos de un cierto punto, entonces la conjunción de ambas se satisface para los elementos del entorno de radio más pequeño, porque este conjunto está contenido en el otro.

El estudiante 2 presenta en sus apuntes un elemento destacable: en sus registros alude, varias veces, al recíproco de un teorema, tanto al referirse al tema de límites como en otros, sobre todo en los casos en que éste era falso.

En esto podría reconocerse la intención del docente de contribuir a que los estudiantes construyan una idea del condicional en la que se distingan como diferentes los roles del antecedente y del consecuente y, por lo tanto, la diferencia entre condición necesaria y condición suficiente. Posiblemente se pretenda, así, evitar que los estudiantes incurran en la llamada falacia abductiva en la que el condicional es mirado como si en realidad fuera un bicondicional; tal como la refiere Crespo (2007), en esta forma de razonamiento (incorrecta desde el punto de vista de la Lógica) se concluye la premisa a partir del condicional y de la 
conclusión, es decir, de (A) $\rightarrow$ (B) y B se obtiene A, donde A y B son fbf.

\subsubsection{Análisis de textos}

En todos los casos, se tomaron textos usuales en los cursos en estudio, que usan tanto profesores como estudiantes.

Para el caso de Cálculo se revisaron Edwards y Penney (2008) y Stewart (1999).

El texto de Edwards y Penney (2008) tiene una organización que incluye al final de cada sección una lista de ejercicios, un cuestionario de ítems falso-verdadero y una lista de preguntas sobre elementos conceptuales. No manifiesta, de manera expresa, la preocupación por resaltar las estructuras lógicas utilizadas, pero en algunas de estas listas o cuestionarios aparecen tareas en las que puede reconocerse la intención de proponer actividades en esta dirección, como se destaca a continuación.

Al tratar la idea de límite, en la sección 2.2, comienza por expresar la noción en forma coloquial, enuncia ciertos teoremas que se refieren a cálculo de límites (a los que llama leyes, entre las que se cuentan una versión del teorema de límites para funciones compuestas) y más adelante, en la sección 2.3, presenta la definición formal, reservando a un anexo, el D, las demostraciones de algunos teoremas.

En una de las preguntas conceptuales, al final de la sección 2.2, propone la construcción de un ejemplo de funciones $f$ y $g$ tales que $\lim _{x \rightarrow a} f(x)=b \quad y \quad \lim _{x \rightarrow b} g(x)=c$ pero $\lim _{x \rightarrow a} g(f(x)) \neq g\left(\lim _{x \rightarrow a} f(x)\right)$ y pide comentar por qué este resultado no contradice el teorema enunciado sobre límites de funciones compuestas: "Si $\lim _{x \rightarrow a} f(x)=b$ y $\lim _{x \rightarrow b} g(x)=c$ entonces $\lim _{\mathrm{x} \rightarrow \mathrm{a}} \mathrm{g}(\mathrm{f}(\mathrm{x}))=g\left(\lim _{\mathrm{x} \rightarrow \mathrm{a}} \mathrm{f}(\mathrm{x})\right) ”($ EDWARDS; PENNEY, 2008, p. 71)

Los autores parecen querer hacer notar que la ausencia de contradicción deriva de que, excepto una, todas las hipótesis del teorema de límites para funciones compuestas son satisfechas en el ejemplo que se pide construir, mientras que no se satisface lo enunciado en la tesis. Es importante que los estudiantes reconozcan que la aplicación de un teorema a un caso concreto de cálculo depende de que todas las hipótesis del teorema se verifiquen en la situación en estudio, para poder afirmar que ocurre lo asegurado en la tesis.

Sin embargo, al no complementar el ejercicio pidiendo proponer la construcción de un ejemplo en el que no se satisfaga alguna de las hipótesis y aun así, se cumpla lo anunciado en 
la tesis, podría favorecerse en los estudiantes la idea de que las hipótesis no son sólo una condición suficiente sino también necesaria, incurriendo en una falacia abductiva.

Continuando con el análisis, Edwards y Penney (2008) dan, en la sección 2.3, la definición formal en términos de $\varepsilon$ - $\delta$ : "el número L es el límite de $f(x)$ cuando $x$ tiende a $a$, y se puede escribir $\lim _{x \rightarrow a} f(x)=L$, considerando que se satisface el siguiente criterio: dado cualquier $\varepsilon>0$ exista un número correspondiente $\delta>0$ tal que $|f(x)-L|<\varepsilon$ para toda $\mathrm{x}$ tal que $0<\mid x$ - $a \mid<\delta$ (20)" (EDWARDS; PENNEY, 2008, p. 85, destaques de los autores).

En esta instancia, se nota la preocupación por explicitar que la sentencia “ $|\mathrm{f}(\mathrm{x})-\mathrm{L}|<$ $\varepsilon$ para toda $\mathrm{x}$ tal que $0<|\mathrm{x}-\mathrm{a}|<\delta$ " (EDWARDS; PENNEY, 2008, p. 85) expresa un condicional, que puede traducirse por si ' $0<\mathrm{x}-\mathrm{a} \mid<\delta$ entonces $|\mathrm{f}(\mathrm{x})-\mathrm{L}|<\varepsilon$ ' o bien por ' $0<$ $|\mathrm{x}-\mathrm{a}|<\delta$ implica que $|\mathrm{f}(\mathrm{x})-\mathrm{L}|<\varepsilon^{\prime}$.

Posteriormente, se muestra un especial cuidado por aclarar que en todos los casos debe encontrarse un valor positivo para $\delta$, una vez se haya tomado uno para $\varepsilon$. Con esto se atiende a la necesidad de destacar las instancias de cuantificación presentes en la definición (una, la de $\varepsilon$, universal y la otra, la de $\delta$, existencial). Sin embargo, no se procede igual en las dos últimas formas de expresar la sentencia (20), en las que no hay mención alguna al hecho de que para cualquier valor de $\mathrm{x}$ debe satisfacerse el condicional.

El texto de Stewart (1999) no tiene alusiones explícitas a las formas deductivas que utiliza. En particular, al abordar la definición de límite, en la sección 2.2, se inclina por una informal, expresada en lenguaje coloquial y basada en la noción de distancia entre números reales. En la sección 2.3 enumera una lista de teoremas sobre cálculo de límites, comenta que todas ellos pueden demostrarse formalmente y ejemplifica cómo podrían utilizarse para hallar límites.

Reserva la formalidad al apéndice $\mathrm{D}$, en el que da la definición habitual en términos de $\varepsilon$ y $\delta$, pero no presta atención a las instancias de cuantificación. Usa esta definición para demostrar que una cierta función tiene límite y en el siguiente apéndice, el E, prueba el teorema sobre límite de la suma, en medio de otras demostraciones.

En los ejemplos en los que se muestra el uso de las leyes de los límites para calcular, se nota la preocupación por hacer evidente que es necesario verificar las hipótesis para poder obtener la conclusión. Sin embargo, en las dos ocasiones en las que esto no ocurre, la conclusión que se brinda al estudiante es que el pretendido límite no existe. Esta es la misma situación referida anteriormente, en relación con el texto de Edwards y Penney (2008).

En suma, en los dos textos revisados no hay señalamientos explícitos a las estructuras 
lógicas utilizadas en las diferentes argumentaciones, y en algún caso, algunos ejemplos podrían, incluso, inducir a la asunción de estructuras deductivas incorrectas.

Para estudiar el tratamiento de estos temas en el curso de Lógica, se seleccionaron dos textos, el de Grimaldi (1997) y el de Rosen (2004).

Grimaldi (1997) titula el segundo capítulo Fundamentos de Lógica y, en él comienza por la construcción de fórmulas (sin dar una definición rigurosa de fórmulas bien formadas), presentando las tablas de verdad. A continuación, presenta la noción de tautología y basa en ella la de equivalencia lógica. A partir de la tercera sección, aborda la implicación lógica y las reglas de inferencia. Su definición se establece a partir de que una cierta sentencia condicional resulta ser una tautología.

En efecto, llama argumento válido a una sentencia $\left(p_{1} \wedge p_{2} \wedge \ldots \wedge p_{n}\right) \rightarrow q$ que sea una tautología. Amplía esta definición diciendo que $p$ implica lógicamente a $q$ (siendo $p$ y $q$ fórmulas cualesquiera), cuando $p \rightarrow q$ es una tautología, y usa para representar esta situación la notación, $p \Rightarrow q$. Una vez establecidas las reglas de inferencia, las usa para proveer ejemplos de pruebas construidas a partir de conjuntos de premisas, con lo que cierra el tratamiento del cálculo proposicional.

Inicia el estudio del cálculo de predicados a partir de la descripción de la necesidad de introducir cuantificadores para ampliar el alcance del cálculo proposicional. Llama proposiciones abiertas a aquellas sentencias en las que se puede reconocer la presencia de variables individuales, y explica de qué forma se ven afectadas mediante la introducción de un cuantificador. A partir de aqui, sigue un camino similar al recorrido antes: aborda la noción de valor de verdad de una proposición abierta, plantea la noción de equivalencia entre proposiciones, presenta las reglas de inferencia (extendiendo las vistas para el cálculo proposicional para considerar las nuevas que aparecen al trabajar con cuantificadores) y cierra el capítulo con la construcción de pruebas a partir de premisas.

En todo el desarrollo se percibe una inclinación a acercar el uso del lenguaje natural al específicamente lógico, mediante la traducción de argumentaciones coloquiales a la exactitud de fórmulas para representarlas, y por advertir acerca de prácticas usuales que implican riesgos de error en su uso.

Vale la pena destacar un comentario en el que Grimaldi (1997) alerta acerca de la confusión en el uso del condicional y del bicondicional, que ya se ha mencionado. Atribuye esa confusión al hecho de que en el uso del idioma frecuentemente se utilizan condicionales para describir situaciones que, por su propia naturaleza, constituyen casos en los que se da una 
equivalencia.

Más adelante, plantea la situación simétrica de ésta, al enfatizar que las definiciones en Matemática son la expresión de una equivalencia y, por tanto, deberían ser formuladas como un bicondicional, cosa que habitualmente no ocurre en los libros de texto, en los que se recurre a una formulación condicional para estas cuestiones.

A eso se agrega la preocupación por ilustrar con aplicaciones en otras áreas de Matemática, donde se pueden resaltar dos ejemplos.

El primero refiere, específicamente, a la definición de límite, destacando la presencia de los cuantificadores en el proceso de escribir la negación de que una cierta función tenga un cierto límite en un punto.

El segundo presenta el enunciado de la propiedad asociativa de una ley de composición interna, comentando que su formulación requiere el uso de cuantificadores, y que este hecho es, frecuentemente, olvidado en el tratamiento de esta propiedad en los textos, lo que debería ser advertido por los profesores a los estudiantes, para ayudarles a reconocer instancias en las que en forma oculta se están usando cuantificadores.

En la exposición de Grimaldi (1997) se reconoce el propósito de explicitar aspectos del uso de sentencias condicionales (distinción entre un enunciado condicional y una equivalencia, diferenciación de los roles del antecedente y del consecuente, papel de los cuantificadores), además de resaltar los procesos de traducción entre el lenguaje natural y el formal, y la manera en que los conceptos estudiados aparecen tratados en libros de otras ramas de Matemática.

El texto de Rosen (2004) propone en su primer capítulo el estudio de Lógica, y en particular, de la demostración (junto con el de conjuntos y funciones). Elige la misma aproximación que el de Grimaldi (1997), llamando proposiciones a cualquier sentencia susceptible de ser clasificable como cierta o falsa. Introduce los conectivos lógicos y las tablas de verdad, para, a partir de allí, presentar las nociones de tautología y de equivalencia. Hace menciones concretas a las formas de traducir entre el lenguaje natural y el formal, tanto aquí como en Cálculo de Predicados, que desarrolla con la presentación de los cuantificadores, y avanza mostrando cómo construir fórmulas complejas y calcular sus valores de verdad.

Sobre esta base dedica una sección a los procesos de demostración, a partir de la introducción de reglas de inferencia (que se presentan a través de tautologías cuyo conectivo principal es un condicional), tanto para el Cálculo Proposicional como para el de Predicados.

En todo este proceso se distinguen instancias en las que se busca aproximar el uso de los elementos desarrollados en otras áreas, tanto en Matemática (la definición de límite se 
presenta como ejemplo de fórmula con cuantificadores anidados) como en Ciencias de la Computación.

En general, el desarrollo de Rosen (2004) es menos profundo que el de Grimaldi (1997). En particular, no se encuentran advertencias acerca de errores o inexactitudes en los tratamientos cotidianos del condicional o de otros conectivos, ni tampoco alusiones al uso dado en otros textos de Matemática a estos instrumentos.

\subsection{La Experimentación}

Interesó a este estudio indagar el efecto que, sobre estudiantes ingresantes a la universidad, tuvieron los cursos universitarios iniciales de Cálculo y Matemática Discreta, para facilitar el uso de estructuras deductivas de uso frecuente en razonamientos o argumentaciones. Además, se quiso averiguar si los efectos eran mayores en los cursos que incorporaban la enseñanza explícita de temas de Lógica como parte de sus contenidos.

\subsubsection{Diseño y aplicación de la experimentación}

Se decidió trabajar con estudiantes ingresantes a carreras de la Facultad de Ingeniería y Tecnologías (FIT) de la Universidad Católica del Uruguay (UCU); en Informática (en adelante grupo I) fueron los trece integrantes que completaron todas las instancias (doce de ellos hombres), por un lado, y por otro, en Electrónica, Telecomunicaciones, Industrial o Alimentos (grupo $\mathrm{O}$ ) con veinte integrantes finales (catorce de ellos hombres).

Se procedió así pues la carrera en Informática era la única de la FIT que tenía en su currículo un curso de Matemática Discreta en el primer semestre, y a diferencia de las restantes, no tenía curso de Cálculo Diferencial en ese período. Esta separación permitía contar con dos grupos de estudiantes con la característica de que ningún alumno estaba en ambos, uno de los cuales (grupo I) recibiría enseñanza específica en temas de Lógica, incluyendo Cálculo Proposicional y Reglas de Inferencia, mientras que el otro (grupo O) tendría una aproximación a estos asuntos a partir de su uso en demostraciones o razonamientos deductivos propios de la estructuración conceptual del Cálculo Diferencial. Ambos cursos se desarrollaron en el turno vespertino, con clases entre las 18:00 y las 22:00 horas.

Las dos asignaturas correspondían al mismo semestre de la respectiva carrera (el primero) y tenían la misma carga horaria semanal de cuatro módulos de ochenta minutos, que 
se ofrecían en dos días de la semana en bloques de dos módulos separados por un recreo de diez minutos.

El diseño fue cuasi-experimental pre-post con una variable independiente de grupo con dos modalidades (curso de Informática y de Electrónica) y como variable dependiente de test inicial - test final una prueba de análisis de conocimientos matemáticos.

El test inicial se usó con dos fines: para tener un indicador del grado de conocimiento de los ingresantes sobre inferencia y sentencias condicionales; y, para establecer una comparación inicial inter grupos, I-O.

El test final se usó, también, para dos propósitos: para comparar estos grupos, I-O al final del semestre; y, para comparar cada grupo consigo mismo y detectar eventuales diferencias de desempeño entre el comienzo y el final del semestre.

La composición del test inicial se realizó de la siguiente manera.

Todos los estudiantes ingresantes a la FIT participan de una prueba diagnóstica al ingreso, compuesta por ítems de múltiple opción con cuatro distractores.

Para esta ocasión, se incluyeron en este cuestionario seis ítems sobre temas de inferencia y formas deductivas. Se comentan algunos en el análisis de los resultados.

Cuatro de estos ítems presentaron, de modo coloquial, argumentos sobre cuestiones cotidianas (MOROU; KALOSPYROS, 2011). Los otros dos interrogaron acerca de contenidos matemáticos (divisibilidad, imágenes y preimágenes de funciones). En forma deliberada, en algunos de ellos la respuesta correcta dependió de reconocer que una sentencia condicional es cierta cuando el antecedente es falso (DURAND-GUERRIER, 2003).

Se asignó a cada estudiante la calificación dada por el promedio de respuestas correctas en estos seis ítems. El conjunto de estas calificaciones constituye el indicador inicial. En la sección que sigue, se presentan algunos de estos ítems y se efectúa un análisis didáctico de ellos.

El test final se construyó de una manera más compleja. Teniendo en cuenta las características de cada curso, se elaboraron preguntas a ser incluidas en las respectivas instancias de evaluación, sobre contenidos propios de cada uno. Esta decisión obligó a tener en consideración dos cuestiones: la de la semejanza de estas preguntas con las del test inicial, por un lado, y por otro, la semejanza entre sí de los dos cuestionarios finales.

En este contexto, semejanza debe entenderse en el sentido de que las estructuras deductivas presentes en cada caso fueran las mismas, aun cuando estuvieran aplicadas a contenidos diferentes. En cualquiera de los casos, se utilizó el procedimiento de revisión por pares. Tres profesores eligieron estos ítems de un conjunto elaborado para esta instancia, con 
el criterio de que hubiera unanimidad en cuanto a su semejanza.

En el caso del grupo I, tres de los ítems del test inicial se repitieron en el final, dado que su estructura permitía incluirlos en el curso de Matemática Discreta; en cambio, sólo uno de los del test inicial pudo ser usado en el final del grupo $O$, porque era el único que trataba sobre contenidos de Cálculo.

Las preguntas del test final fueron formuladas en las instancias de evaluación de los respectivos cursos. Esta decisión se tomó para minimizar la interferencia en el desarrollo de los cursos y para mantener el desarrollo habitual de la enseñanza en cada curso.

Para estudiar eventuales diferencias entre los grupos, tanto entre ambos (en el test inicial o el final) como respecto a sí mismos (entre test inicial y final) se usaron tests $\mathrm{t}$ de Student, para muestras independientes, en el primer caso, y relacionadas en el segundo, y complementariamente, las pruebas no paramétricas U de Mann-Whitney (para muestras independientes) y Wilcoxon (para relacionadas). Los resultados de estos análisis se presentan en la sección 3.2.3, a continuación de la que sigue.

\subsubsection{Análisis didáctico}

La Tabla 1 presenta los porcentajes de respuestas correctas registrados en cada uno de los ítems usados en la prueba de diagnóstico.

Tabla 1 - Porcentaje de respuestas correctas registrados en los ítems del test inicial

\begin{tabular}{c|c|r|r|r|r|r}
\hline Ítem & 1 & 2 & 3 & 4 & 5 & 6 \\
\hline Porcentaje & 0,20 & 0,03 & 0,59 & 0,31 & 0,58 & 0,28 \\
\hline
\end{tabular}

Fuente: Prueba de diagnóstico al ingreso a la UCU

Estos ítems resultaron difíciles: ninguno de ellos fue respondido correctamente por más del $60 \%$ de los participantes. Además, están separados en dos clases en base a su dificultad, 1, 2, 4 y 6, por un lado, y 3 y 5, por otro, donde los de la segunda clase casi duplican el resultado más alto de los de la primera.

A continuación, se analizan dos ítems, uno de cada clase, para profundizar en estos aspectos.

El ítem 2, perteneciente al primer grupo y notable por ser el más difícil, es el siguiente ${ }^{2}$ :

Se hace la siguiente afirmación sobre los números enteros mayores que 0 y

${ }^{2}$ Adaptado de Durand-Guerrier (2003) 
menores que 10:

"Si $n$ es un número impar, entonces $n+1$ es múltiplo de 3"

Esta afirmación es cierta:

a) Sólo para $n=2, n=4, n=5, n=6$ y $n=8$.

b) Sólo para $n=5$.

c) Sólo para $n=2, n=5$ y $n=8$.

d) Sólo para $n=1$ y $n=5$.

En este ítem, la opción correcta es la a) y para señalarlo así es necesario reconocer que para 2, 4,6 y 8 el antecedente ( $n$ es impar) resulta falso, por lo que la sentencia es cierta, además de que para $n=5$, tanto antecedente como consecuente ( $n+1$ es múltiplo de 3") son ciertos.

La respuesta que registró mayor porcentaje de aciertos fue la b); en este distractor tanto el antecedente como el consecuente son ciertos para $n=5$. Esto parece indicar que los valores 2, 4, 6 y 8 no se consideraron relevantes en el argumento para quienes la eligieron, porque para ellos es falso el antecedente.

La respuesta c) registró el segundo mayor porcentaje. Los que la consideraron correcta parecen haber observado sólo el valor del consecuente, que resulta cierto sólo para los tres naturales indicados en el antecedente.

La respuesta d) obtuvo un porcentaje de respuesta tan bajo como el de la a). En este caso, el antecedente resulta cierto tanto para 1 como para 5, pero para 1 el consecuente es falso. Podría conjeturarse que esto significa que se reconoce que el condicional es falso cuando se da esta combinación de valores en antecedente y consecuente.

El ítem 5, correspondiente al segundo grupo, se formuló como sigue:

Se dan las siguientes afirmaciones:

Llueve o está frío. No llueve. Si no llueve y está frío, nieva.

Si estas afirmaciones son ciertas a la vez, es posible concluir que:

a) Alguien se moja.

b) No está frío.

c) Llueve.

d) Nieva

La respuesta correcta, que es la d), puede obtenerse deductivamente de las premisas mediante, por ejemplo, la siguiente secuencia:

i) Del par Llueve o está frío y No llueve se obtiene la conclusión parcial Está frío.

ii) Se tiene, por lo tanto, que No llueve y está frío al conjuntar la conclusión anterior con la segunda de las premisas.

iii) Del par No llueve y está frío y Si no llueve y está frío, nieva. Se obtiene Nieva.

Aunque las reglas de inferencia usadas no se expliciten, habitualmente aparecen en las argumentaciones que se realizan en demostraciones, por lo que son más próximas a la 
experiencia de los estudiantes que la noción, mucho más técnica, de que un condicional sólo es falso cuando su antecedente es cierto y su consecuente falso.

La respuesta a) recibe un porcentaje de respuestas sorprendentemente alto, del $20 \%$, pese a que la afirmación que se hace en ella no guarda relación alguna con las premisas.

La respuesta b), donde lo afirmado es la negación de la consecuencia de las dos primeras premisas descrita antes, recibe el segundo porcentaje más bajo de respuestas, en tanto la respuesta c), donde se pone como consecuencia la negación de una de las premisas, fue la que tuvo menor porcentaje de respuestas. Esto podría indicar que se reconoció su falsedad a partir de algún argumento deductivo.

Un modelo para la evaluación de la comprensión de una prueba (MEJÍA-RAMOS et al., 2012) plantea como uno de los pasos a dar el de la justificación de afirmaciones, es decir, cómo se concluye una cierta afimación de otras hechas con anterioridad (HODDS; ALCOCK; INGLIS, 2014). La familiariad de los estudiantes con estos procesos podría explicar el elevado procentaje de aciertos en este ítem.

El ítem restante del segundo grupo se refiere a una relación causal. El relativo buen desempeño en este ítem puede estar relacionado con la asociación causalidad-sentencia condicional ya señalada.

La siguiente pregunta fue planteada en el examen del curso de Matemática Discreta, y formó parte del postest, como uno de los ítems propuestos sobre los contenidos del curso.

Considere la siguiente matriz $\boldsymbol{A}$ que representa una relación $R$ definida en el conjunto $X=\left\{x_{1}, x_{2}, x_{3}, x_{4}, x_{5}\right\}$

$$
\mathbf{A}=\left(\begin{array}{lllll}
1 & 0 & 0 & 0 & 0 \\
0 & 1 & 0 & 0 & 0 \\
0 & 1 & 1 & 1 & 0 \\
0 & 0 & 0 & 0 & 0 \\
1 & 0 & 0 & 0 & 1
\end{array}\right)
$$

Indique cuál de las siguientes afirmaciones es falsa:

a) El par $\left(x_{4}, x_{4}\right)$ no pertenece a $R$; por eso, $R$ no verifica la propiedad idéntica.

b) No existen elementos $x_{i}, x_{j}$ y $x_{k}$ tales que $\left(x_{i}, x_{j}\right)$ y $\left(x_{j}, x_{k}\right)$ están en $R$ y $\left(x_{i}, x_{k}\right)$ no está en $R$; por lo tanto, $R$ posee la propiedad transitiva.

En ambos casos la respuesta es afirmativa, aunque por motivos diferentes. Las dos afirmaciones refieren a definiciones en cuya formulación aparecen cuantificadores. En el caso de la b), el no cumplimiento de la sentencia para un caso, alcanza para afirmar la no validez de la propiedad recíproca o simétrica. Pero en el caso de la propiedad transitiva, además aparece un condicional, que resulta cierto porque en todos los casos el antecedente es falso.

Para el postest correspondiente al curso de Cálculo, se repitió el ítem, ya usado en el pretest (que figura como ítem 4 en la tabla 1), que se da a continuación. 
La gráfica que se da es la de una función $f$ definida en el intervalo $\{x /-4 \leq x \leq 4\}$.

Indica cuál de las siguientes afirmaciones sobre la función f es falsa:

a) Si $0 \leq f(x) \leq 1$, entonces $-4 \leq x \leq 0$.

b) Si $-3 \leq x \leq 0$, entonces $0 \leq f(x) \leq 2$.

c) Si $f(x)>1$ entonces $x<0$.

d) Si $1<x \leq 4$ entonces $f(x)>-2$.

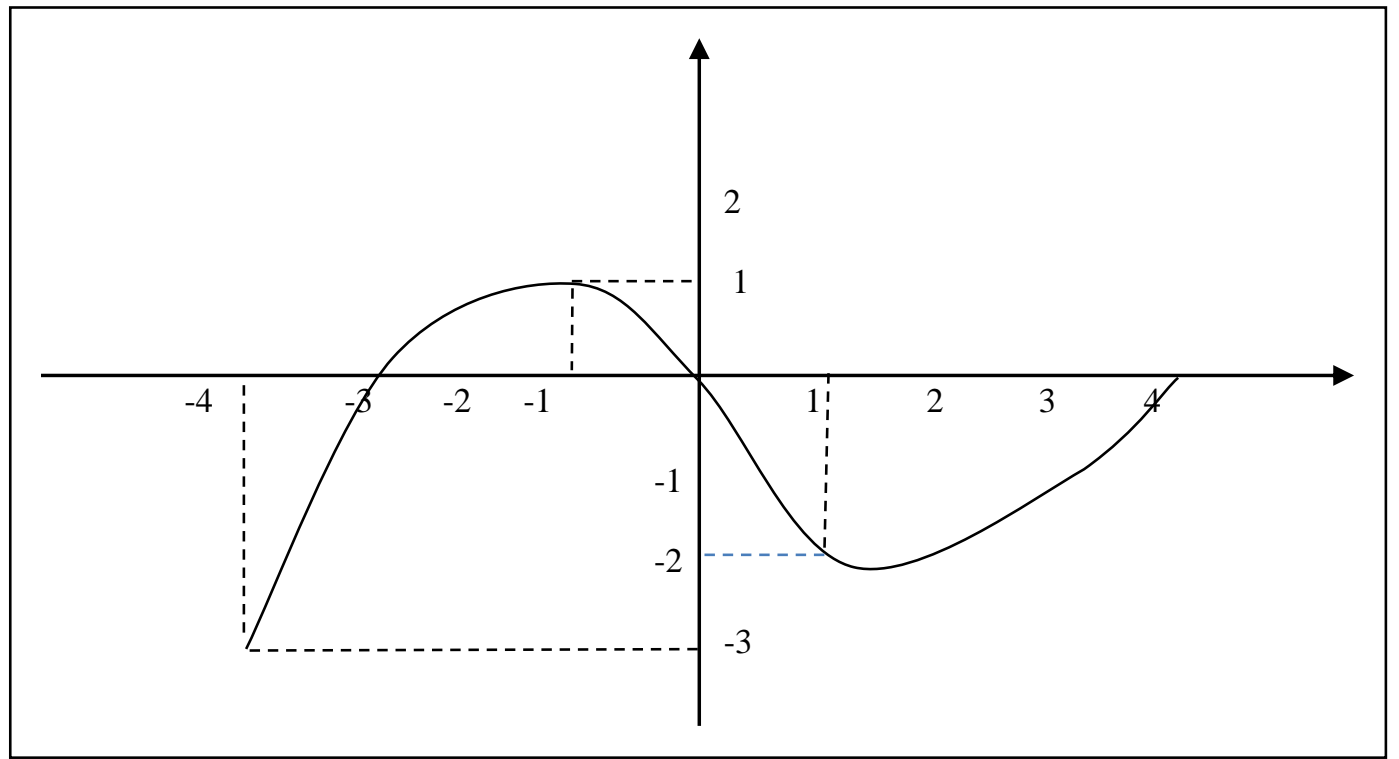

Figura correspondiente al ítem 4 de la Prueba de diagnóstico al ingreso a la UCU

La respuesta es la parte d), ya que es la única sentencia falsa. La parte a) presenta una sentencia fácilmente identificable como cierta. La de la parte b), siendo cierta, presenta una dificultad adicional y es que en el consecuente no se da exactamente la imagen del intervalo que figura en el antecedente, sino uno que lo contiene. Pero el más difícil de reconocer como cierto es el enunciado de la parte c), porque en él el antecedente es falso.

Este análisis permite conjeturar que los estudiantes reconocen mejor cadenas deductivas como las usuales en demostraciones matemáticas que cuestiones más técnicas como la definición del valor de verdad del condicional.

En efecto, los ítems referidos en la Tabla 1 responden a dos grupos: el que integran las preguntas 1, 2, 4 y 6 (que son las de más bajo porcentaje de respuestas correctas) y el formado por las preguntas 3 y 5 (cuyo porcentaje de respuestas correctas casi duplica cualquiera de los anteriores).

En los ítems del primer grupo la opción correcta se obtiene a partir del hecho de que una sentencia condicional es cierta si su antecedente es falso. Y, en los ítems donde se registró el mejor resultado, la respuesta correcta puede obtenerse por argumentos que son más próximos a los que se usan habitualmente en la clase de Matemática. 


\subsubsection{Análisis de los resultados: comparaciones entre grupos}

Además de constituir una necesidad metodológica, establecer las condiciones iniciales de los dos grupos respondía a otra cuestión adicional. Las pruebas de diagnóstico al ingreso en la FIT se vienen proponiendo hace años, y en los últimos se detecta una tendencia que marca diferencias significativas en los desempeños de los estudiantes desagregados por carrera, siendo los de Informática los que obtienen el menor. Interesó saber si esta situación se repetía al considerar sólo los ítems que formaban parte del test inicial. Como muestra la Tabla 2, éste fue el caso.

Tabla 2 - Comparación de medias iniciales y nivel de significación

\begin{tabular}{c|c|c|c|r}
\hline Grupo & Media & $\begin{array}{c}\text { Desviación } \\
\text { típica }\end{array}$ & $\begin{array}{r}\mathrm{t} \\
\text { de Student }\end{array}$ & $\begin{array}{r}\text { U de } \\
\text { Mann-Whitney }\end{array}$ \\
\hline I & 0,36 & 0,18941 & 00,008 & 0,005 \\
\hline O & 0,57 & 0,21171 & \\
\hline
\end{tabular}

Fuente: Prueba de diagnóstico al ingreso a la UCU

Este dato debe tenerse en consideración al vincular los resultados del pretest con los del postest. La diferencia se compensa, y el grupo de Matemática Discreta presenta una media mayor. La Tabla 3 compara los desempeños finales de los estudiantes en las dos materias, muestra que las diferencias no son significativas, lo que marca una equiparación al terminar los cursos.

Tabla 3 - Comparación de medias finales y nivel de significación

\begin{tabular}{c|c|c|r|r}
\hline Grupo & Media & $\begin{array}{c}\text { Desviación } \\
\text { típica }\end{array}$ & $\begin{array}{r}\text { t de } \\
\text { Student }\end{array}$ & $\begin{array}{r}\text { U de } \\
\text { Mann-Whitney }\end{array}$ \\
\hline I & 0,58 & 0,16315 & 0,12 & 0,09 \\
\hline O & 0,44 & 0,27409 & 09 & 0 \\
\hline
\end{tabular}

Fuente: Resultados del Postest

Este primer resultado permite plantear que la enseñanza intencional en temas de Lógica que recibieron los estudiantes del grupo de Matemática Discreta fue suficiente para que mejoraran su desempeño y equipararan, al menos, al del grupo de Cálculo.

Esta interpretación se ve reforzada cuando se comparan los grupos en relación a sí mismos, como muestra la Tabla 4.

Tabla 4 - Comparación de medias inicial y final y niveles de significación

\begin{tabular}{|c|c|c|c|c|c|c|}
\hline $\begin{array}{ll} & \text { Gru } \\
\text { po } & \end{array}$ & Media inicial & $\begin{array}{l}\text { Desviación } \\
\text { típica }\end{array}$ & Media final & $\begin{array}{l}\text { Desviación } \\
\text { típica }\end{array}$ & $\begin{array}{l}\mathrm{T} \\
\text { de Student }\end{array}$ & Wilcoxon \\
\hline I & 0,36 & 0,18941 & 0,58 & 0,16315 & 0,007 & 0,01 \\
\hline $\mathrm{O}$ & 0,57 & 0,21171 & 0,44 & 0,27409 & 0,69 & 0,93 \\
\hline
\end{tabular}

Fuente: Prueba de diagnóstico al ingreso a la UCU y resultados del Postest

Aquí, se evidencian una mejora muy significativa en el desempeño del grupo de Matemática Discreta y una estabilidad en el de Cálculo. 
Otros resultados se dan a continuación, basados en la consideración de los ítems presentados en la sección anterior.

En el caso del ítem que integró tanto el pretest como el postest del grupo I (referido como ítem 1 de la Tabla 1), el porcentaje de respuestas correctas pasó de $0,14^{3}$ a 0,64. Esta mejora informa que el número de estudiantes que respondieron correctamente al finalizar el curso más que cuadruplicó el de los que lo hicieron al comienzo, y es un fuerte apoyo a la explicación dada.

El otro ítem referido al curso de Matemática Discreta (propuesto en el examen de la asignatura), si bien registró valores más bajos de respuestas correctas que el anterior $(0,54$ y 0,46 , respectivamente, para las partes b) y d)) también supera a los resultados del pretest en los ítems semejantes a éste.

En cambio, cuando se revisan los resultados del ítem referido al curso de Cálculo, los registros son más bajos. Para el ítem analizado antes, que también formó parte del cuestionario final de este curso (que figura como ítem 4 en la Tabla 1), se registraron resultados similares en las dos instancias, 0,28 y 0,27 respectivamente. Un dato interesante es que el error más frecuente es señalar como correcta a c): el distractor que presentaba una sentencia correcta por ser falso el antecedente. Esta recibe más aceptación que la respuesta correcta, 0,31 y 0,35 en los test inicial y final respectivamente.

\section{Conclusiones y reflexiones finales}

Los resultados recogidos en el estudio realizado muestran que los estudiantes ingresan a la universidad con conocimientos escasos acerca de las estructuras deductivas para comprender o construir razonamientos que aparecen con frecuencia en los cursos que deben encarar a su ingreso, lo que resulta alineado con las investigaciones reseñadas por DurandGuerrier (2008) y Camacho, Sánchez-Pozos y Zubieta (2014).

En particular, que una sentencia condicional resulta cierta cuando su antecedente es falso, independiente del valor de verdad del consecuente, parece ser casi ignorado por los estudiantes. En este sentido, apuntan los desempeños del diagnóstico y los del postest de Cálculo analizados.

Este hecho tiene notable importancia, porque en varias instancias con las que los estudiantes deberán tomar contacto en sus cursos universitarios, se utiliza este resultado (entre

${ }^{3}$ El resultado de 0,20 que figura en la Tabla 4 para este ítem incluye a todos los estudiantes y no sólo a los de Informática. 
otros, la prueba de que el conjunto vacío es subconjunto de cualquier otro, o la de que el conjunto vacío es linealmente independiente, o la necesidad de que el punto donde se calcula el límite de una función sea de acumulación del dominio).

A partir del estudio previo, examinando los apuntes acopiados por estudiantes, y analizando los textos de Cálculo de uso frecuente, no se encuentran evidencias de enseñanza intencional de temas de Lógica en los cursos de Cálculo de bachillerato o universidad.

En otro orden, la sola exposición de los estudiantes al uso de formas de razonamiento deductivo no parece tener efecto en su adquisición, como emerge de la consideración de los resultados del curso de Cálculo, en especial de los que refieren al ítem usado tanto en el pretest como en el postest de esta asignatura; en este no se constata mejora en el porcentaje de respuestas correctas entre las dos instancias.

Este hecho obliga a poner en cuestión modos de pensar que afirman la existencia de una correlación positiva entre la enseñanza de la Matemática y los aprendizajes de formas de razonamiento de esta disciplina. Por eso, el tema de la presencia de referencias explícitas desde la enseñanza a las formas deductivas usadas en Matemática requiere ser indagado específicamente.

En contrapartida, el estudio del curso de Matemática Discreta muestra que la enseñanza intencional de estos contenidos parece tener efectos positivos sobre su adquisición y uso. Estos resultados concuerdan con los que reportan Morou y Kalospyros (2011) o Hodds, Alcock y Inglis (2014).

En otro orden, una pregunta a responder es si es necesario encomendar a un curso específico, como el de Matemática Discreta, la enseñanza de estos temas. En este sentido, Durand-Guerrier (2005) presenta argumentos para sostener que es importante la explicitación de las estructuras lógicas (en particular, la presencia de cuantificadores) en los procesos de demostración, siguiendo los procedimientos de deducción basados en reglas de inferencia.

Sin embargo, muchas carreras universitarias no incluyen, en sus diseños curriculares, espacios para el tratamiento de estos contenidos. Por eso, resulta interesante seguir indagando acerca de qué intervenciones didácticas, en el marco de cursos universitarios del área (Cálculo, Álgebra Lineal, Probabilidad, Estadística, entre otros) pueden resultar exitosas para propiciar en los estudiantes aprendizajes y desarrollo de habilidades en el uso de estructuras deductivas, tanto en áreas científicas como de formación profesional.

\section{Referencias}


ARCE SÁNCHEZ, M.; CONEJO GARROTE, L.; ORTEGA DEL RINCÓN, T. ¿Cómo son los apuntes de matemáticas de un estudiante? Influencia de los elementos matemáticos y sus relaciones. Enseñanza de las Ciencias, España, v. 1, n. 34, p. 149-172, 2016.

AMADO, N.; SÁNCHEZ, J.; PINTO, J. A. Utilização do Geogebra na Demonstração Matemática em Sala de Aula: o estudo da reta de Euler. BOLEMA, Rio Claro, v. 29, n. 52, p. 637-657, 2015.

BLOCH, I. Teaching Functions on a Graphic Milieu: What Forms of Knwolegde Enable Students to Conjecture and Prove? Educational Studies in Mathematics, Holanda, v. 52, p. 3-28, 2003.

BRUNSCHVICG, L. Las etapas de la filosofía matemática. Buenos Aires: Lautaro, 1945.

CAMACHO, V.; SÁNCHEZ POZOS, J. J.; ZUBIETA, G. Los estudiantes de ciencias, ¿pueden reconocer los argumentos lógicos involucrados en una demostración? Enseñanza de las Ciencias, España, v. 1, n. 32, p. 117-138, 2014.

CRESPO, C. Las argumentaciones matemáticas desde la visión de la socioepistemología. México, D.F.: Centro de Investigación en Ciencia Aplicada y Tecnología Avanzada (CICATA), 2007.

CRESPO, C.; FARFÁN, R.; LEZAMA, J. Argumentaciones y demostraciones, una visión de la influencia de los escenarios socioculturales. Revista Latinoamericana de Matemática Educativa, México, v. 3, n. 13, p. 283-306, 2010.

DÍAZ, L. Concepciones en el aprendizaje del concepto de límite: un estudio de casos. Santiago de Chile: Pontificia Universidad Católica de Chile, Facultad de Educación, 1999.

DURAND-GUERRIER, V. Wich notion of implication is the right one? From logical considerations to a didactic perspective. Educational Studies in Mathematics, Holanda, n. 53, p. 5-34, 2003.

DURAND-GUERRIER, V. Natural deduction in predicate calculus a tool for analysing proof in a didactic perspective. In: Congress of the European Society for Research in Mathematics Education, 4., 2005, España. Proceedings of the Fourth Congress of the European Society for Research in Mathematics Education. Marianna Bosch (Editor), 2005. p. 409-419.

DURAND-GUERRIER, V. About logic, language and reasoning at the transition between French upper Secondary school and University. In: ICME, 9., 2008, Praga. Proceedings of the Ninth Congress of the European Society for Research in Mathematics Education. Konrad Krainer y Nad'a Vondrová (Editores), 2008. p. 409-419.

EDWARDS, C.; PENNEY, D. Cálculo con trascendentes tempranas. México: Prentice-Hall, 2008.

GLYMOUR, C. Thinking Things Through: An Introduction to Philosophical Issues and Achievements. Massachusetts: The MIT Press, 1997.

GRIMALDI, R. Matemáticas Discreta y Combinatoria, una introducción con aplicaciones. Wilmington: Addison Wesley Iberoamericana, 1997.

HODDS, M.; ALCOCK, L.;INGLIS, M. Self-Explanation Training Improves Proof Comprehension. Journal For Research in Mathematics Education, Estados Unidos, v. 1, n. 45, p. 62-101, 2014.

INGLIS, M.; ALCOCK, L. Experts and Novices Approaches to Reading Mathematical Proofs. Journal for Research in Mathematics Education, Estados Unidos, v. 4, n. 43, p. 358-390, 2012.

INGLIS, M.; SIMPSON, A. Conditional inference and advanced mathematical study. Educational Studies in Mathematics, Holanda, n. 67, p. 187-204, 2008. 
KLINE, M. El pensamiento matemático de la antigüedad a nuestros días. Madrid: Alianza Editorial, 1972.

MEJÍA-RAMOS, J. P. et al. An assessment model for proof comprehension in undergraduate mathematics. Educational Studies in Mathematics, Holanda, v. 1, n.79, p. 3-18, 2012.

MOROU, A.; KALOSPYROS, N. The role of logic in teaching, learning and analyzing proof. : CONGRESS OF THE EUROPEAN SOCIETY FOR RESEARCH IN MATHEMATICS EDUCATION, 7., 2011, Working Group 1. Disponible en http://www.cerme7.univ.rzeszow.pl/WG/1/CERME7_WG1_Morou\&Kalospyros.pdf. Acceso el 25 ago. 2018.

PEANO, G. Sur la Definition de la Limite d'une Fonction. Exercice de Logique Mathématique. , American Journal of Mathematics, Estados Unidos, v. 1, n. 17, p. 37-68, 1895.

ROSEN, K. Matemática discreta y sus aplicaciones. Madrid: Mc Graw Hill, 2004.

STEWART, J. Cálculo, conceptos y contextos. México: International Thomson Editores, 1999.

Submetido em 24 de Julho de 2017. Aprovado em 20 de Abril de 2018. 